Research article

\title{
Inhalation of Sodium Pyruvate to Reduce the Symptoms and Severity of Respiratory Diseases Including COVID-19, Long COVID, and Pulmonary Fibrosis
}

\author{
Christopher R. Lupfer ${ }^{1}$, Riley Nadler ${ }^{1}$, Ronald Amen², Alain Martin ${ }^{* 2}$ \\ 1.Missouri State University, Springfield, MO, USA
}

2.EmphyCorp/Cellular sciences inc. Flemington, NJ, USA

Corresponding author: Dr. Alain Martin, EmphyCorp/Cellular sciences inc, Flemington, NJ 08822, USA

Received: October 04, 2021; Accepted: October 26, 2021; Published: October 29, 2021

\begin{abstract}
Background: To combat the continuing COVID-19 pandemic, and to treat the symptoms in Long COVID patients safe, effective, and inexpensive treatments are needed. Patients recovering from severe COVID-19 are at serious risk of developing pulmonary fibrosis. Conversely, patients with pulmonary fibrosis have an increased risk and susceptibility to COVID-19 infection, demonstrating the need to treat both. Design: Three separate clinical trials were conducted 1) in COVID-19 infected patients, 2) in Long COVID patients, and 3) in patients with Pulmonary Fibrosis to determine the efficacy of N115, a sodium pyruvate based nasal spray. Patient symptoms, vital signs and respiratory function were evaluated compared to a placebo control or a no treatment baseline control. Findings: During active COVID-19 infection, N115 decreased viral titers and produced a significant improvement over saline in coughing/sneezing and fatigue. In Long COVID patients, N115 significantly reduced headache, coughing/sneezing and increased $\mathrm{SaO}^{2}$ levels (decreased hypoxemia) and improved breathing (dyspnea). In patients with Pulmonary Fibrosis, there was a significant improvement in all lung functions, compared to baseline, as determined by changes in SaO2, FVC, FEV1, PEF, and FEV1/FVC ratio. Conclusions: N115 is safe and effective at reducing symptoms of active COVID-19 infection and improves disease condition in Long COVID patients. Furthermore, N115 significantly improves lung function in Pulmonary Fibrosis patients. As COVID-19 and Pulmonary Fibrosis are associated with each other, our clinical research demonstrates that N115 is a promising treatment for both and adds to the current 19 human clinical trials where N115 has shown efficacy in thousands of patients, regardless of the etiology of the lung disease (COPD, CF, allergic rhinitis, sinusitis, the flu, COVID-19 infected patients, Long COVID and patients with Pulmonary Fibrosis).
\end{abstract}

Keywords: Pyruvate, COVID-19, long COVID, pulmonary fibrosis, immune modulator

\section{Introduction}

COVID19 is a disease caused by the novel SARS-CoV-2 virus [1]. In the last 1.5 years since the spread of this virus began a world-wide pandemic, hundreds of millions of people have become infected and millions have died [2]. Although the advent of any novel pathogen is likely to result in widespread infection and mortality, SARS-CoV-2 induces a particularly severe and rapid form of pneumonia in some patients concomitant with an overall cytokine storm but diminished interferon responses [3]. Although case severity varies by sex, age and comorbidities, some of the most severe comorbidities include high blood pressure, diabetes and interstitial lung disease [4].
Interstitial lung disease encompasses a large group of chronic lung disorders associated with excessive tissue remodeling, scarring, fibrosis, decreased FEV1 values, decreased $\mathrm{SaO} 2$ and decreased Nitric Oxide (NO) associated with nasal inflammation that causes congestion, coughing and sleep disorders $[5,6]$. Researchers have demonstrated that pulmonary fibrosis increases risk and susceptibility to COVID-19 infection [7]. Acute exacerbations of idiopathic pulmonary fibrosis (IPF) are known as serious events, which can reach a mortality rate of $50 \%$ when viral infections play a role [8]. This isn't surprising considering pulmonary fibrosis and severe cases of COVID-19 share a few 
common risk factors, including: increasing age, male sex, diabetes and hypertension [9, 10]. Given that pulmonary fibrosis (PF) debilitates lung function, it makes sense that PF would only increase the risk of having a severe case of COVID-19.

Understandably so, these overlapping risk factors are cause for concern when it comes to mitigating a double attack on the lungs, should a patient become exposed to COVID-19. Inversely, people recovering from severe COVID-19 are at serious risk of developing PF $[9,10]$ clearly demonstrating the two-way relationship between COVID-19 and PF, which calls for specific considerations in how they interact. There are millions of patients worldwide with Long COVID symptoms, (patients that had COVID with lingering symptoms), including coughing, fatigue, low $\mathrm{SaO} 2$, and many with respiratory issues like $\mathrm{PF}$ and interstitial lung disease [8,11]. In 2015, there were thousands of complaints to the FDA stating that steroids, and all the available nasal spray products, failed to provide relief from nasal inflammation or treat the symptoms of IPF [12]. With many Long COVID patients developing PF and interstitial lung disease, new therapies are needed.

In the COVID-19 infection arm of this research study, we show that N115 is slightly better than saline at reducing viral loads, but N115 was clinically superior over saline in reducing some symptoms of COVID-19 infections, including coughing/sneezing and fatigue. In long COVID, N115 significantly reduced hypoxemia (low SaO2), coughing/sneezing, trouble breathing, and headaches. In our current and ongoing clinical trials examining the effects of sodium pyruvate nasal spray (N115), we discovered that patients with PF with COPD and IPF without a COPD component experienced significant improvement, including less coughing, improved nasal irritation/erythema, increased average expelled-NO, higher $\mathrm{SaO} 2$, and improved lung function (FVC, FEV1, PEF, and FEV1: FVC ratio).

\section{Methodology}

\section{COVID-19 animal research}

Animal research was conducted at the Regional Biocontainment Laboratory at the University of Tennessee Health Sciences Center, Memphis, TN under Institutional Animal Care and Use Committee (IACUC) protocol 2021.013A according to IACUC guidelines, AVMA Guidelines on Euthanasia, NIH regulations (Guide for the Care and Use of Laboratory Animals), and the U.S. Animal Welfare Act of 1966. Two groups of ten 5-6 weeks old female K18-hACE2 transgenic mice were infected by intranasal installation of $800 \mathrm{pfu}$ of SARS CoV-2 P3 isolate USAWA1/2020 in $50 \mu 1$ saline. Mice were then treated from day 0-9 with nebulized saline (control) or N115 3x daily for 30 minutes each treatment. Mice were weighed and monitored for survival daily for 14 days.

\section{COVID-19 infected clinical trial}

Prior to conducting human clinical trials, IRB approval was obtained (CIRBI:Pro00049340) and the trials were registered on www.clinicaltrials.gov (NCT04824365, NCT04871815). The study protocol was prepared in accordance with the revised Helsinki Declaration for Biomedical Research Involving Human Subjects and Guidelines for Good Clinical Practice and patients included in this study were provided written informed consent.
This was a two-phase study. In the first phase, thirty adults with confirmed active COVID-19 infections (by qRT-PCR) were randomly, and blindly, assigned to either a saline nasal spray or a saline + sodium pyruvate nasal spray (N115) treatment group. Patients were instructed to use their spray $3 \mathrm{x}$ daily for 14 days. Patient's vital signs (BP, SaO2, HR, RR, Temp.) were monitored and nasal swabs tested for SARS-CoV-2 levels every 2 days for 14 days. Patients were asked to complete a Daily Symptoms Log every day for 14 days, scoring the symptoms on a Likert scale from 0 -10 with 10 representing the most severe symptoms. Symptoms included fatigue, coughing/sneezing, sore throat, chills, congestion, trouble breathing, headache and body ache. Patients also recorded their body temperature $2 \mathrm{x}$ daily for the 14 days.

\section{Long COVID clinical trial}

In the second phase of the study, 22 patients were enrolled and served as their own negative controls. Patient's vital signs (BP, $\mathrm{SaO} 2, \mathrm{HR}, \mathrm{RR}$, Temp.) were recorded on the first day, and patients were asked to complete daily symptoms log every day for 7 days without the use of the study medication. Patient's symptoms (coughing/sneezing, chills, trouble breathing, body aches, headaches, fatigue, anxiety, loss of taste/smell and sore throat) were scored on the log using a Likert scale from 0-10 with 10 representing the most severe symptoms. On day 8 , patient's vital signs were recorded again as a baseline and the patient administered the first dose of N115. Fifteen minutes later, the patient's vital signs were again tested, and the patients were asked to complete the same daily symptoms log every day for 7 more days while using N115 3x daily as a nasal spray. After the second week, the patients returned for a final collection of vital signs.

\section{Pulmonary fibrosis clinical trial}

An initial twenty-one-day sub-chronic clinical trial was conducted that included fifteen patients with PF (9 with PF and COPD and 6 with IPF without COPD) that remained on their normal medications but were also administered the $20 \mathrm{mM}$ sodium pyruvate nasal spray (N115). If the patients were also on nasal sprays as part of their normal therapy, that nasal spray was eliminated. In all 15 patients, the test results were compared to their previous three-week screening and baseline data on their current therapies as the baseline control for each variable for all their lung functions (FEV1, FVC, PEF, FEV1/FVC ratios, SaO2, Nitric oxide, coughing rates, and nasal inflammation). Following this, five new patients with PF and COPD had their medications removed and were administered N115 for three days in order to assess its effect without any other medication.

\section{Results}

\section{COVID-19: Acute infections in animals}

Previously, we demonstrated that treatment with sodium pyruvate can improve inflammation and decrease viral loads in mice during infection with influenza A virus and HSV1 [13,14]. As pyruvate acts on the host immune response, through metabolic pathways and not directly on the virus [15], our data demonstrate that sodium pyruvate is a promising treatment option that is safe, effective, and unlikely to elicit antiviral resistance. We, therefore, examined the effects of N115 treatment in hACE2 transgenic mice infected with SARS-CoV-2 to determine safety and efficacy.

Mice treated with nebulized N115 lost significantly less weight 


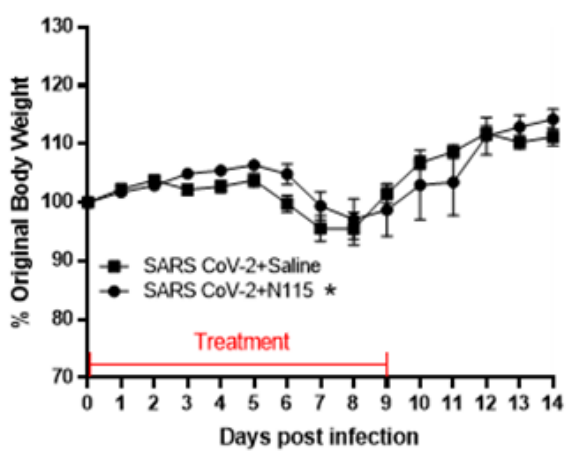

Figure 1. Effects of N115 treatment in mice infected with SARS CoV-2. Two groups of ten mice each were infected with 800pfu of SARS CoV-2 P3 isolate USA-WA1/2020 in 50 $\mu$ saline intranasally. One group of ten mice was then treated with PBS 3x daily and the other group of ten mice treated with N115 3x daily. Mice were weighed daily and monitored for malaise and mortality for 14 days. Statistical analysis was performed by two-way ANOVA $\left({ }^{*} \mathrm{p}<0.05\right)$.

compared to mice treated with nebulized saline (Figure 1). Mortality was similar between groups, but the infectious dose was not expected to result in high mortality. From this preliminary animal study, and in conjunction with multitudes of previously reported safety data $[13,14]$, we proceeded with a clinical trial in humans with active infection of SARS CoV-2.

\section{COVID-19 infected patients}

This clinical trial was designed to determine the safety and efficacy of N115 against saline in COVID-19 infected patients. Thirty adults (Demographics presented in Table 1) with confirmed (positive RT-qPCR test) active COVID-19 infections were randomly, and double blindly, assigned to either a saline nasal spray or a saline $+20 \mathrm{mM}$ sodium pyruvate nasal spray
(N115) treatment. Patients self-administered the sprays 3x daily for 14 days. Saline is acknowledged (Edenborough ELVIS project) to physically reduce nasal viral titers by $0.5 \operatorname{logs}$ to $0.7 \operatorname{logs}$ over untreated patients and reduces mucus and allergens which subsequently reduces congestion, trouble breathing, and sore throats [16]. Therefore, saline is not a true placebo for this study but a standard of care. Viral titers in N115 treated patients were lower compared to saline treated patients through day 8 as measured by RT-qPCR from nasal swabs $(\mathrm{p}<0.0197)$ (Figure 2A). N115 lowered viral titers below 10,000, the value that has been reported to significantly decrease transmission of the virus [17]. The mean day for patients to drop below 10,000 viral genome copies as measured by RT-qPCR from nasal swabs was day 6.4 for N115 vs. day 7.7 for saline.

Patient's vital signs (BP, SaO2, HR, RR, Temp.) were monitored every 2 days for 14 days. Patients were asked to complete a Daily Symptoms Log every day for 14 days, scoring the symptoms on a Likert scale from 0-10 with 10 representing the most severe symptoms. Patients also recorded their body temperature $2 \mathrm{x}$ daily for the 14 days. Over the fourteen-day trial, there was no significant change in blood pressure (BP), heart rate (HR), or respiratory rate (RR). We observed similar improvements in patients treated 3x daily with either saline or N115 in SaO2, trouble breathing, and sore throat (Figure 2B and Table 1). However, N115 performed significantly better with coughing/sneezing $(p<0.0435)$ and fatigue $(p<0.0001)$ symptoms over saline (Figure $2 \mathrm{C}-\mathrm{D}$ and Table 1). We observed significant improvements in patients treated $3 \mathrm{x}$ daily with either saline or N115 over the 14 days, for fever, body aches, headaches and chills that resolved and returned to normal levels by day 14 as viral numbers decreased, but conversely, N115 treatment resulted in higher body temperature (Fever, $\mathrm{p}<0.0030)$ and higher scores for body aches $(p<0.0001)$, headaches $(p<0.0001)$, and chills $(p<0.0001)$ over saline (Figure 2E-H and Table 1). No adverse events were re-

Table 1. Patient Demographics and Symptom Data.

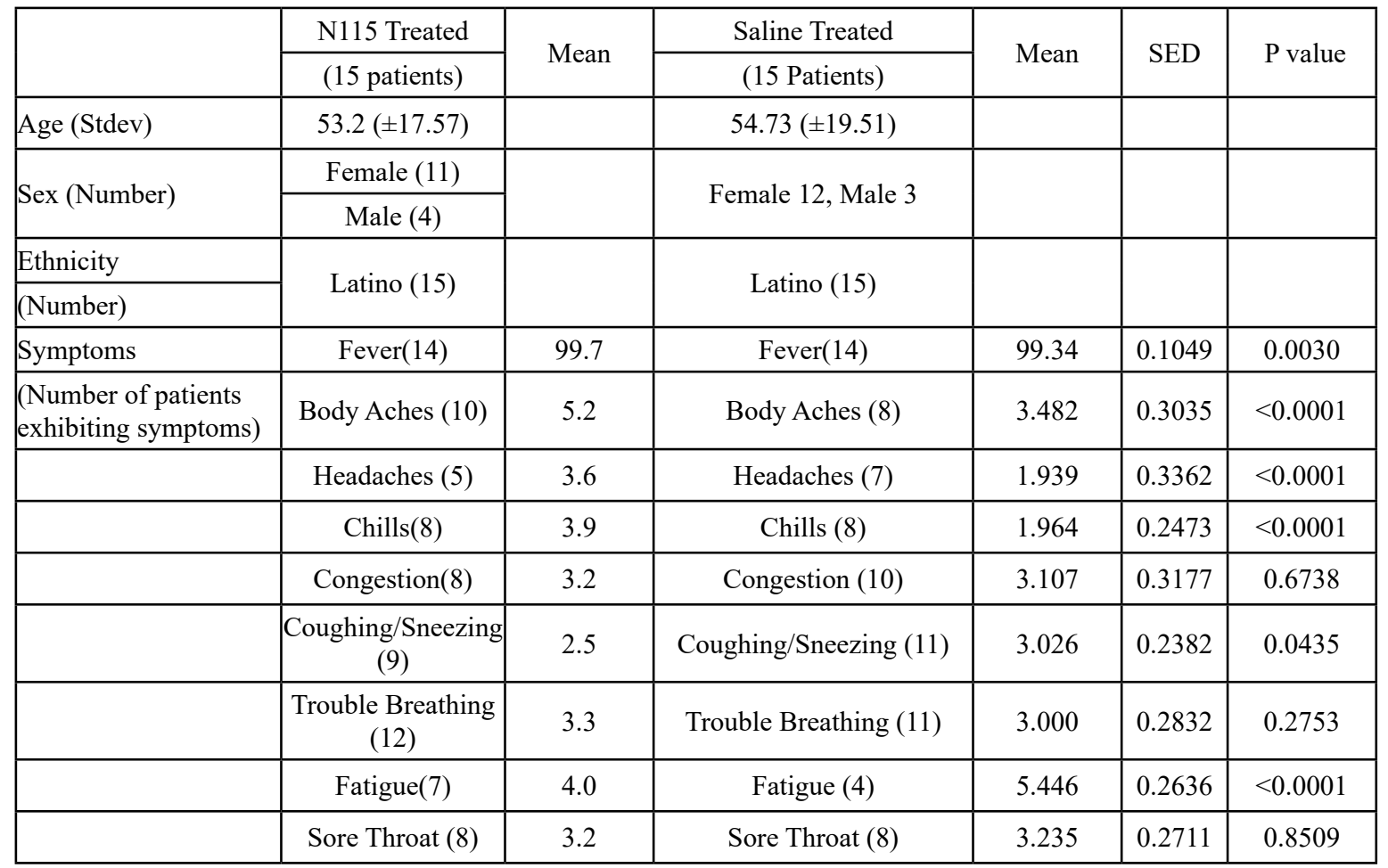



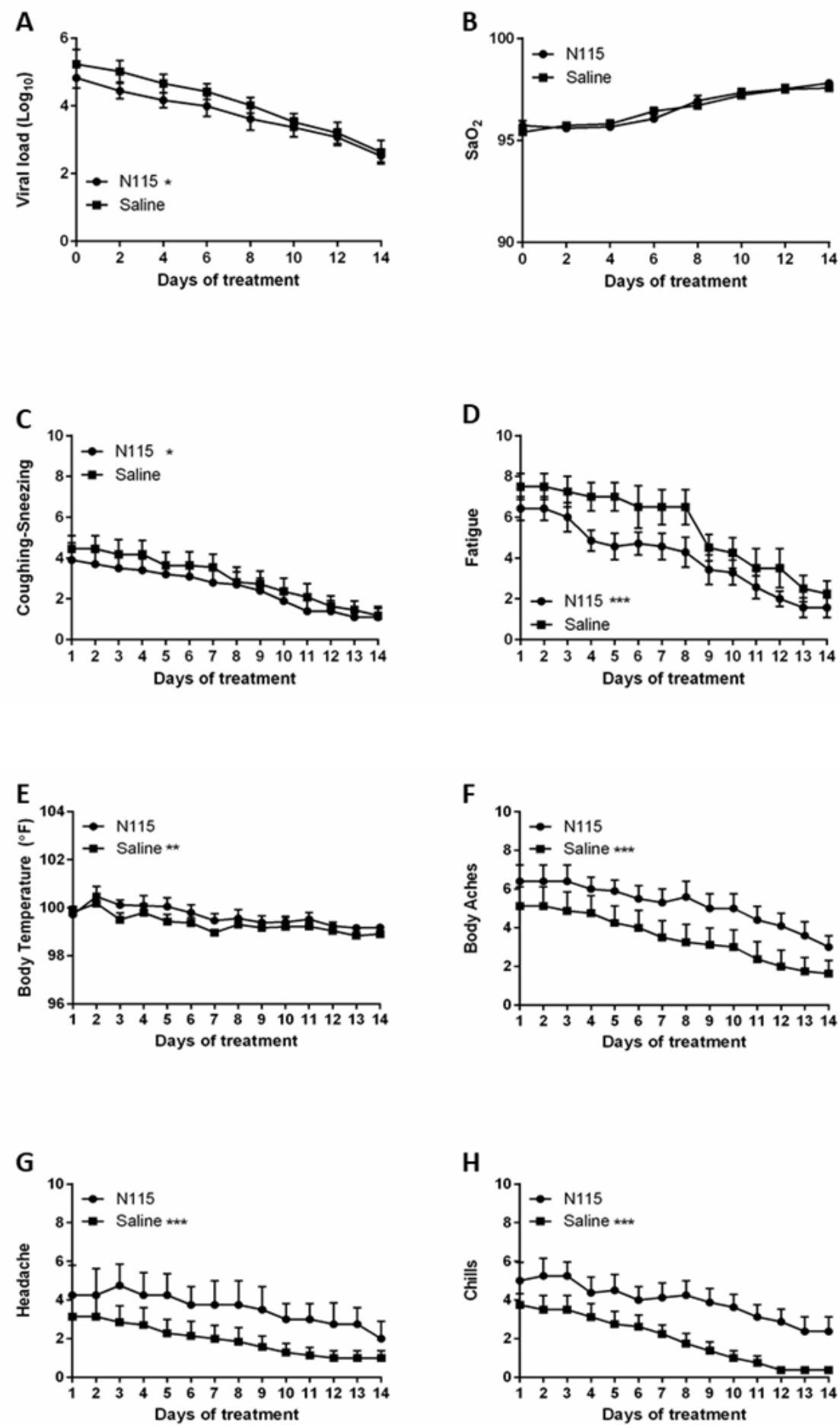

Figure 2. Effects of N115 treatment in Active COVID-19 Infection.

Thirty patients were randomly assigned to either the saline control or the N115 treatment group. Viral titers (A) and $\mathrm{SaO} 2$ (B) were measured every 2 days. Coughing (C), Fatigue (D), Fever (E), Body Aches (F), Headaches $(\mathrm{G})$, and Chills $(\mathrm{H})$ were measured or scored daily on a Likert Scale $(0-10,10=$ most severe). Data were analyzed for statistical significance by two-way ANOVA. $\left({ }^{*} \mathrm{p}<0.05, * * \mathrm{p}<0.01, * * * \mathrm{p}<0.0001\right)$.

ported from the use of either saline or N115 by patients or clinical staff.

\section{Long COVID}

We next examined the effects of N115 in patients that were experiencing long-term symptoms after recovering from active COVID-19. These patients, known as Long-COVID patients, were monitored for symptoms for one week with no treatment followed by one week of treatment with N115. Patients were not randomized but served as their own negative controls. During the initial 7 days when patients received no treatment, there was no significant change in $\mathrm{SaO} 2$ or heart rate, but there was a slight improvement in BP $1.25 \mathrm{mmHg}(\mathrm{p}=0.015)$ (Figure 3AD). Heart rate remained stable throughout the trial (Figure $3 \mathrm{~B}$ ). There was additional improvement in BP after N115 treatment within 15 minutes after the first treatment (Day 8 post vs. Day 1, $-2.25 \mathrm{mmHg}, \mathrm{p}<0.0001$ ) and BP remained lower on day 14 (Day 14 vs Day $1,-2.0 \mathrm{mmHg}, \mathrm{p}=0.0026$ ) (Figure 3C-D). Importantly, 15 minutes after the first dose of N115 was administered on day $8, \mathrm{SaO} 2$ improved by $0.5 \%$ from the pretreatment reading on the same day $(\mathrm{p}=0.0114)$. It continued to improve, and on day 14 , $\mathrm{SaO} 2$ levels improved by $1.63 \%$ over day 1 and $1.5 \%$ over day 8 
A

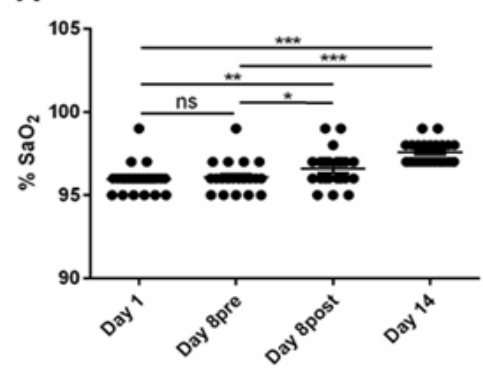

C

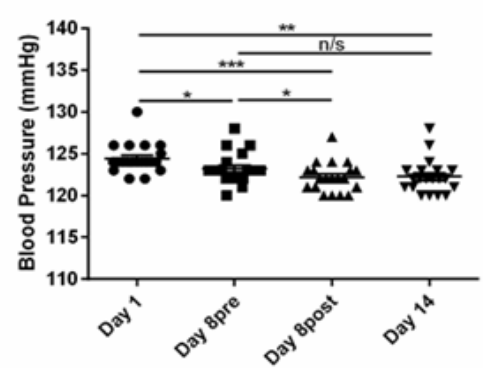

E

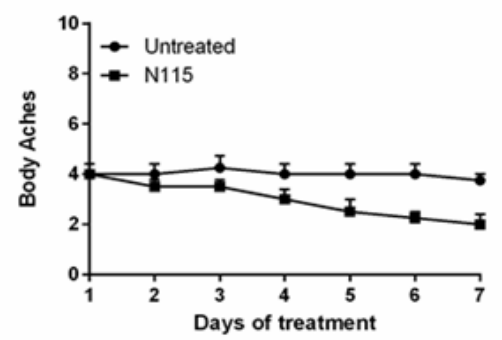

G

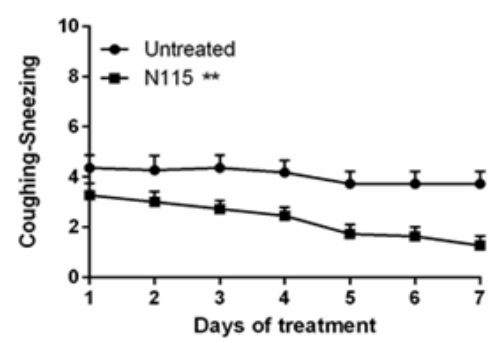

B

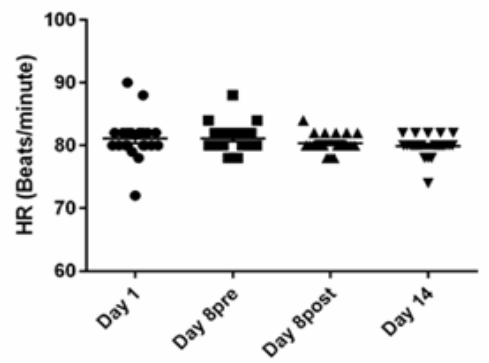

D

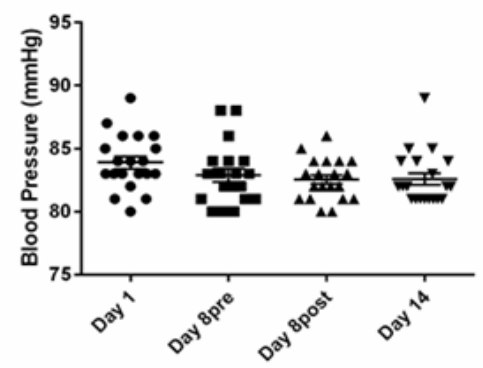

$\mathbf{F}$

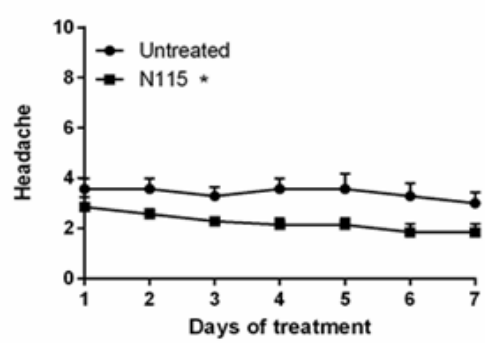

H

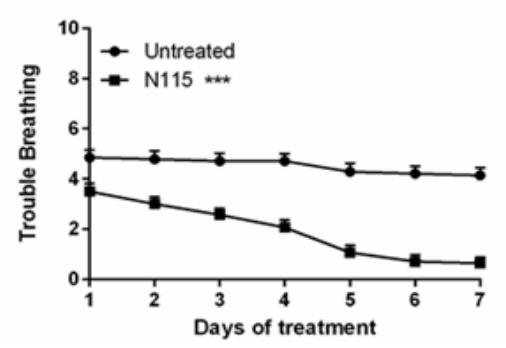

Figure 3. Results from N115 treatment of Long COVID Patients.

Twenty-two patients were monitored for signs and symptoms for 7 days without treatment and then for an addition 7 days with N115 treatment. SaO2 (A), Heart Rate (B), Systolic Blood Pressure (C) and Diastolic Blood Pressure (D) were measured on day 1, day 8 before treatment, day 8 after treatment (15 minutes after treatment), and day 14 (7 days of treatment). All symptoms including Body Aches (E), Headaches $(\mathrm{F})$, Coughing/Sneezing $(\mathrm{G})$ and Trouble Breathing $(\mathrm{H})$ were measured or scored daily on a Likert Scale $(0-10,10=$ most severe $)$ for 7 days prior to treatment and measured again from day 8-14 with N115 treatment. Data were analyzed for statistical significance by oneway ANOVA (A-D) or two-way ANOVA (E-H). $\left({ }^{*} \mathrm{p}<0.05,{ }^{* *} \mathrm{p}<0.01, * * * \mathrm{p}<0.0001\right)$

pretreatment $(\mathrm{p}<0.0001$ and $\mathrm{p}<0.0001)$ (Figure 3A).

During the first 7 days, when there was no treatment, patients reported little to no change in symptoms including body ache, headache, coughing/sneezing, and trouble breathing. However, after N115 treatment for 7 days, patients reported a significant 1.143 point improvement in headaches $(p=0.0373)$, a 2.455 point improvement in coughing/sneezing $(\mathrm{p}=0.0091)$, and a 3.5 point improvement in trouble breathing $(\mathrm{p}<0.0001)$ (Figure $3 \mathrm{E}-$ $\mathrm{H})$. Fatigue, anxiety, loss of taste/smell, congestion and body aches also showed some improvement, but the changes were not significant due to a lack of power from too few patients presenting with these symptoms enrolling in the study (Table 2). Overall, our results demonstrate that N115 significantly improves respiratory function in as little as 15 minutes with substantial improvement within 7 days compared to no treatment controls.

\section{Pulmonary fibrosis}

Many long COVID patients develop PF $(9,10)$. Therefore, we include here our data on N115 treatment of PF. Treatment of 15 patients with pulmonary fibrosis with N115, in addition to their standard medication, resulted in a significant $(p=0.010)$ 
Table 2. Patient Demographics and Symptom prevalence.

\begin{tabular}{|c|c|c|}
\hline \multirow{2}{*}{ Age } & (22 patients) & \\
\hline \multirow{2}{*}{ Sex } & $31.68( \pm 9.25)$ & \\
\hline & Female $(11)$ & \multirow{2}{*}{$(1)$} \\
\hline & Male (11) & \multirow{2}{*}{$(3)$} \\
\hline \multirow{2}{*}{$\begin{array}{c}\text { Sumber of patients exhibit- } \\
\text { ing symptoms })\end{array}$} & Fever $\left(<99.5^{\circ} \mathrm{F}\right)$ & \\
\hline & Hedy Aches & $(7)$ \\
\hline & Chills & $(0)$ \\
\hline & Congestion & $(4)$ \\
\hline & Coughing/Sneezing & $(11)$ \\
\hline & Trouble Breathing & $(16)$ \\
\hline & Fatigue & $(3)$ \\
\hline & Sore Throat & $(1)$ \\
\hline & Smell/ Taste & $(4)$ \\
\hline & Anxiety & $(2)$ \\
\hline
\end{tabular}

A

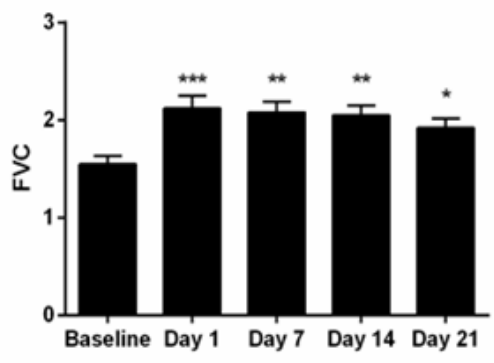

C

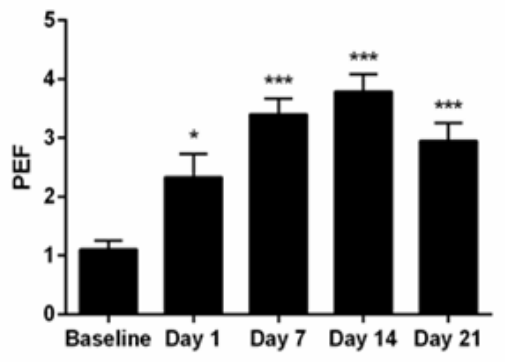

E

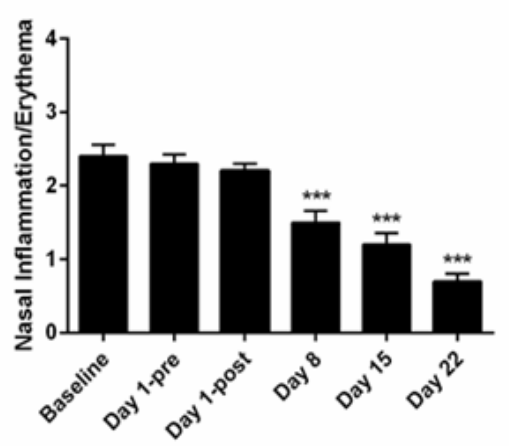

improvement in lung function (breathing) in all patients with IPF without COPD by day eight, and further increasing by day 22 compared to baseline ( $\mathrm{p}=0.0005)$, as determined by changes in FVC, FEV1, PEF, and FEV1/FVC ratios (Figure 4A-C). The improved FEV1/FVC ratio from $52 \%$ to $86 \%$ was clinically significant. N115 treatment also showed that coughing was significantly reduced in all patients $(\mathrm{p}=0.007)$ (Figure 4D), a significant improvement in nasal irritation/erythema with most patients being free of irritation by day $22(\mathrm{p}=0.0001)$ (Figure 4E), and a significant increase in the group average expelled NO by day 8 $(p=0.010)($ Figure 4F). These results indicated that current therapies in use are inadequate alone to treat patient with IPF.

In a second round, five patients with PF and COPD had their medications removed and were administered only N115 nasal spray solution for three days in order to assess its effects. The data from the three-day trial indicated a statistically and clinically significant improvement in lung function compared to baseline with increases that ranged from $12.0 \%$ to $43 \%$ in FVC, FEV1, PEF, and FEV1/FVC ratios (Figure 5A-C). A significant improvement was also seen in $\mathrm{SaO} 2$ levels, compared to base-

B

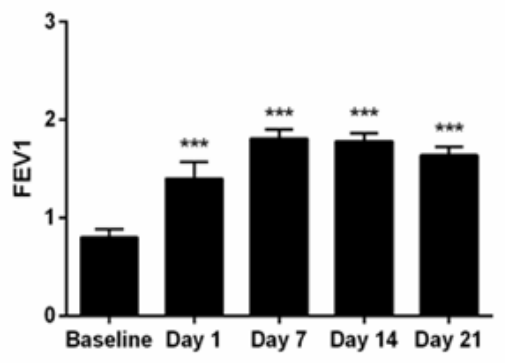

D

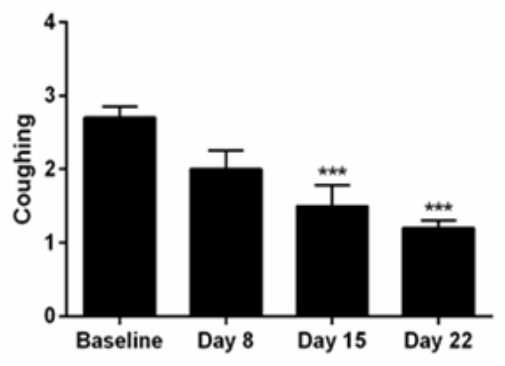

$\mathbf{F}$

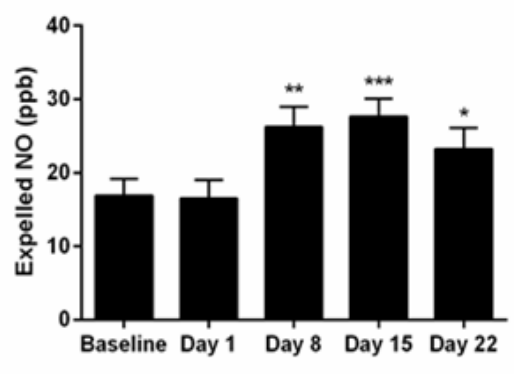

Figure 4. Sub-Chronic treatment of PF patients with N115. 
line, such that all subjects had $\mathrm{SaO} 2$ levels of $\geq 97$, which persisted throughout the trial ( $p$-values $<0.001$ at all time points) (Figure 5D).

\section{Discussion and Conclusions}

To combat the continuing COVID-19 pandemic, and to treat the symptoms in long COVID (hypoxemia (low $\mathrm{SaO} 2$ ), fatigue, coughing/sneezing, trouble breathing, body aches, headaches and pulmonary fibrosis), N115 (sodium pyruvate) was chosen because of its safety and efficacy profile after treating 3.5 million patients in over 200 hospitals globally with no adverse events reported. In 19 Phase I, II, III FDA human clinical trials, against a saline placebo, only N115 reduced inflammation and oxygen radicals and inflammatory cytokines including IL-6, a cause of the cytokine storm in patients with an active COVID-19 infection [18]. In prior clinical trial, N115, not the saline placebo, reduced congestion and coughing while increasing lung functions, increasing the synthesis of $\mathrm{NO}$, and increasing $\mathrm{SaO} 2$ levels in thousands of patients including patients with varying lung diseases like COPD, pulmonary fibrosis, cystic fibrosis, allergic rhinitis, sinusitis and influenza infected patients [18]. Numerous studies have shown oxidative stress to be associated with pulmonary fibrosis, including Long COVID patients with PF, and that antioxidants are effective in attenuating fibroproliferative responses in the lungs of animals and humans [18-22]. Sodium Pyruvate is a natural antioxidant of the human body that inhibits fibrosis and received Orphan Drug Designations for the treatment of Cystic Fibrosis and Pulmonary Fibrosis [14,18,21]. The objective of the clinical trials reported here was to study the safety and efficacy of N115 and changes in lung function and COVID symptoms in acute virally infected COVID-19 patients, patients with chronic symptoms after COVID-19 (Long COVID), and patients with PF.
In the COVID-19 infections study, saline nasal spray was used as a control. However, saline is acknowledged (Edenborough ELVIS project) to physically reduce other Coronavirus titers by 0.5 logs to 0.7 logs over untreated patients, and saline also reduces mucus and allergens which subsequently reduce congestion, trouble breathing, and sore throats [16]. Therefore, saline is not a true placebo. Still, N115 lowered viral titers to below 10,000 by day 6.4 versus day 7.7 for saline. As titers below 10,000 reduce the transmission of COVID-19, this may help decrease virus spread in N115 treated patients [17]. N115 was also significantly better at reducing some of the symptoms of COVID-19 infections including coughing/sneezing and fatigue. Unfortunately, other drugs tested for COVID-19 treatment delivered in saline have reported increased coughing, sore throat, irritation, and other negative symptoms. As reported by the WHO, steroids increased SARS CoV-2 titers over untreated patients, potentially exacerbating infection. Unlike steroids that down-regulate nasal nitric oxide synthesis, sodium pyruvate reduces nasal inflammation while increasing the synthesis of nitric oxide in the nasal passages that is released into the lungs. Nitric oxide is then available to the lungs to fight infections, maintain bronchodilation, increase lung functions and decrease lung fibrosis [23-29].

Over the fourteen-day trial, patients treated with either saline or N115 showed improvement in headaches, trouble breathing, body aches, chills, sore throats, coughing/sneezing and fatigue. N115 treated patients did have slightly higher fever and took longer for chills, body aches and headaches to return to normal. As N115 works by modulating the immune response, including increasing NO etc., and not by direct antiviral activity, some increase in immune responses is anticipated while others are anticipated to decrease [13-15]. However, saline, does not affect in-
A

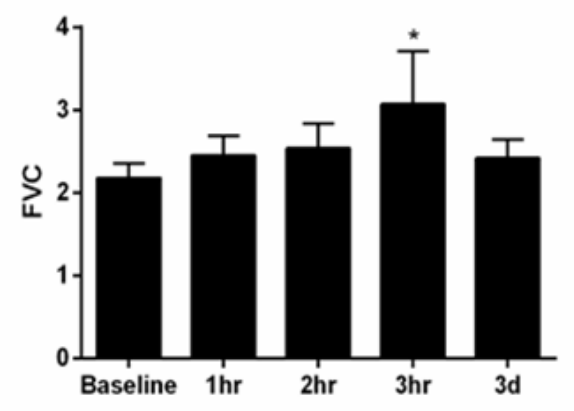

C

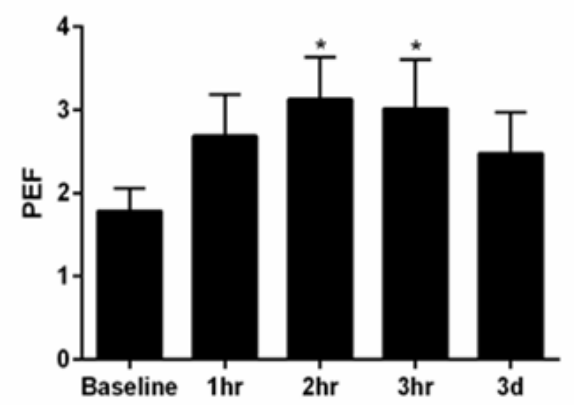

B

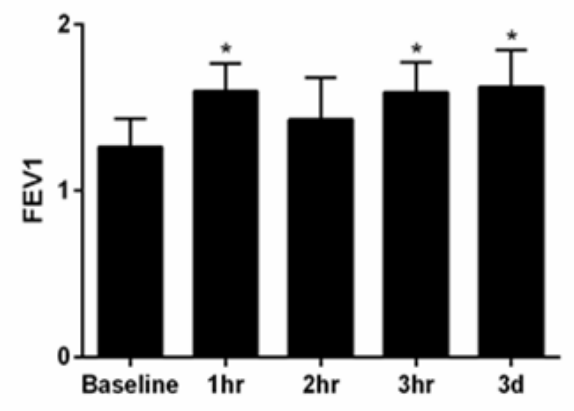

D

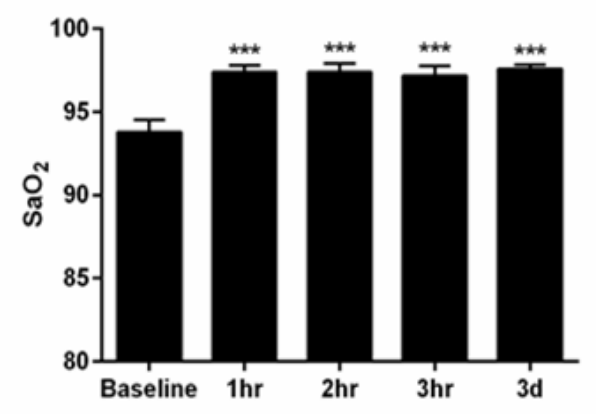

Figure 5. Acute treatment of PF with N115. 
flammation or inflammatory cytokines, does not decrease oxygen radicals, or decrease coughing or increase lung functions, which are all desirable for treatment of COVID-19 and Long COVID or patients with PF and are documented with N115. Furthermore, N115 is not likely to elicit antiviral resistance as it targets the host response and not the virus directly. As SARS CoV-2 variants continue to immerge to the vaccine and are likely to immerge to antiviral drugs, the development of immune modulators like N115 that can treat COVID-19 patients is essential.

Long COVID patients were monitored for symptoms for one week with no treatment followed by one week with treatment with N115. Patients were not randomized but served as their own negative controls. During the first 7 days, when there was no treatment, patients reported little to no change in symptoms. However, after N115 treatment for 7 days, patients reported a significant improvement in headache, coughing/sneezing, and trouble breathing. Most importantly, N115 treatment improved SaO2 from the pretreatment reading on the same day and continued to improve blood oxygenation through day 14 as well as lowering blood pressure. Overall, N115 significantly improves respiratory function, which was supported by the patient's scores on trouble breathing and $\mathrm{SaO} 2$.

Numerous reports indicate that long COVID patients develop pulmonary fibrosis, associated with excessive tissue remodeling, scarring, fibrosis, decreased FEV1 values, decreased $\mathrm{SaO} 2$ and decreased Nitric Oxide (NO) associated with nasal inflammation that causes congestion, coughing, trouble breathing, and sleep disorders [10,11,30-33]. Also, patients with pulmonary fibrosis have an increased risk and susceptibility to COVID-19 infection, which can reach a mortality rate of 50\% [7,34]. Thus, N115 was used to determine its efficacy and safety in these patients too. During the acute treatment of patients with PF with a COPD component, their regular therapy was removed, and they were treated for three days with only N115 nasal spray, which demonstrated a statistically and clinically significant improvement in all lung functions compared to baseline as determined by changes in FVC, FEV1, PEF, and FEV1/FVC ratios, which persisted throughout the three-day trial. A significant immediate average improvement was also seen in $\mathrm{SaO} 2$ levels.

The results in the sub-chronic 21-day clinical test of patients with PF with a COPD component and patients with IPF without a COPD component, showed that inhalation of N115 for 21 days provides a significant reduction in coughing by day eight of the treatment, and continued to decrease over the course of the 21-day treatment. There was a significant improvement in nasal irritation/erythema with most patients being free of irritation by day 22. The group average expelled NO was higher, with 14 of 15 patients showing an increase during the study. Patients with PF with a COPD component remained on their regular therapy. Thus, no improvement in some lung functions was anticipated. However, there was a significant $(\mathrm{p}=0.010)$ improvement in lung function observed in all patients with IPF without COPD, while on their current medications as determined by changes in FVC, FEV1, PEF, and FEV1/FVC ratios. The improved FEV1/FVC ratios from $52 \%$ to $86 \%$ was clinically significant and indicated that current therapies in use are inadequate to treat patient with IPF. This study confirmed the thousands of complaints to the FDA stating that steroids, and all the available nasal spray products, are inadequate to provide relief from nasal inflammation or treat the symptoms of IPF (12). Importantly, very high patient acceptance of the N115 drug was reported (8 to 10 out of 10: "very good" or "excellent").

Although the number of patients enrolled in these trials was small, thus decreasing the power of the statistical analysis, the results all indicate that $\mathrm{N} 115$ is able to improve lung function, especially in patients suffering from chronic conditions such as PF and Long COVID. Seventeen other clinical trials with data submitted to the U.S. FDA also demonstrate similar findings in patients treated with N115 including COPD, cystic fibrosis, allergic rhinitis, sinusitis and influenza infected patients where lung function, $\mathrm{NO}$ and $\mathrm{SaO} 2$ improved with N115 treatment. In this study, as in the previous 17 studies, N115 must be inhaled, and our previous work shows that the route of administration is important for the function of sodium pyruvate $(13,14)$. Overall, our results demonstrate that N115 is a promising treatment that warrants further investigation.

\section{Acknowledgements}

We thank the staff at Family First Medical Research Clinic, Virginia Gardens, FL for patient recruitment and collection of patient data. We also thank Trinity Healthcare and Dynamic DNA Labs, Springfield, MO for collection of patient data.

\section{References}

1. Chen N, M Zhou, X Dong, et al. Epidemiological and clinical characteristics of 99 cases of 2019 novel coronavirus pneumonia in Wuhan, China: a descriptive study. Lancet. 2020; 395: 507-513.

2. Organization WH. Coronavirus disease (COVID-19) Weekly Epidemiological Update and Weekly Operational Update. 2021.

3. Karki R, BR Sharma, S Tuladhar, et al. Synergism of TNF-alpha and IFN-gamma Triggers Inflammatory Cell Death, Tissue Damage, and Mortality in SARS-CoV-2 Infection and Cytokine Shock Syndromes. Cell. 2021 184: 149-168 e117.

4. Williamson EJ, AJWalker, K Bhaskaran, et al. 2020. Factors associated with COVID-19-related death using OpenSAFELY. Nature. 2020; 584: 430-436.

5. Kalchiem-Dekel O, JR Galvin, AP Burke, et al. Interstitial Lung Disease and Pulmonary Fibrosis: A Practical Approach for General Medicine Physicians with Focus on the Medical History. J Clin Med. 2018; 7.

6. Todd NW, IG Luzina, SP Atamas. Molecular and cellular mechanisms of pulmonary fibrosis. Fibrogenesis Tissue Repair. 2012; 5: 11.

7. Naqvi SF, DA Lakhani, AH Sohail, et al. Patients with idiopathic pulmonary fibrosis have poor clinical outcomes with COVID-19 disease: a propensity matched multicentre research network analysis. BMJ Open Respir Res. 2021; 8.

8. Uzel FI, S Iliaz, F Karatas, et al. COVID-19 Pneumonia and Idiopathic Pulmonary Fibrosis: A Novel Combination. Turk Thorac J. 2020; 21: 451-453.

9. Naik PK, BB Moore. Viral infection and aging as cofactors for the development of pulmonary fibrosis. Expert Rev Respir Med. 2010; 4: $759-771$. 
10. Ojo AS, SA Balogun, OT Williams, et al. Pulmonary Fibrosis in COVID-19 Survivors: Predictive Factors and Risk Reduction Strategies. Pulm Med. 2020: 6175964.

11. Groff D, A Sun, AE Ssentongo, et al. Short-term and Long-term Rates of Postacute Sequelae of SARS-CoV-2 Infection: A Systematic Review. JAMA Netw Open. 2021; 4: e2128568.

12. Administration, U. S. F. a. D. 2015. The Voice of the Patient: Idiopathic Pulmonary Fibrosis. 2015.

13. Reel J, C Lupfer. Sodium Pyruvate Ameliorates Influenza A Virus Infection In Vivo. Microbiol Res . 2021;12: 258-267.

14. Sheridan J, E Kern, A Martin, et al. Evaluation of antioxidant healing formulations in topical therapy of experimental cutaneous and genital herpes simplex virus infections. Antiviral Res. 1997; 36: 157-166.

15. Abusalamah H, JM Reel, CR Lupfer, et al. Pyruvate affects inflammatory responses of macrophages during influenza A virus infection. Virus Res. 2020; 286: 198088.

16. Ramalingam S, C Graham, J Dove, et al. Hypertonic saline nasal irrigation and gargling should be considered as a treatment option for COVID-19. J Glob Health. 2020; 10: 010332.

17. Wolfel R, VM Corman, W Guggemos, et al. Virological assessment of hospitalized patients with COVID-2019. Nature. 2020; 581: 465-469.

18. Inc., E. 2021. N115 FDA Submissions.

19. Kelly FJ, A Blomberg, A Frew, et al. Antioxidant kinetics in lung lavage fluid following exposure of humans to nitrogen dioxide. Am J Respir Crit Care Med. 1996; 154: 1700-1705.

20. Mogensen TH, J Melchjorsen, P Hollsberg, et al. Activation of NF-kappa B in virus-infected macrophages is dependent on mitochondrial oxidative stress and intracellular calcium: downstream involvement of the kinases TGF-beta-activated kinase 1, mitogen-activated kinase/extracellular signal-regulated kinase kinase 1, and I kappa B kinase. J Immunol. 2003; 170: 6224-6233.

21. Stanko RT, L O'Hare.The power of pyruvate : the natural way to better health and well being. Keats Pub., Los Angeles, 1999.

22. Suhail S, J Zajac, C Fossum, et al. 2020. Role of Oxidative Stress on SARS-CoV (SARS) and SARS-CoV-2 (COVID-19) Infection: A Review. Protein J. 2020; 39: 644-656.
23. Artlich A, T Busch, K Lewandowski, et al. Childhood asthma: exhaled nitric oxide in relation to clinical symptoms. Eur Respir J. 1999; 13: 1396-1401.

24. Dhar A, JM Brindley, C Stark, et al. Nitric oxide does not mediate but inhibits transformation and tumor phenotype. Mol Cancer Ther. 2003; $2: 1285-1293$.

25. Djupesland PG, JM Chatkin, W Qian, et al. Nitric oxide in the nasal airway: a new dimension in otorhinolaryngology. Am J Otolaryngol. 2001; 22: 19-32.

26. Gallai V, P Sarchielli. Nitric oxide in primary headaches. J Headache Pain. 2000; 1: 145-154.

27. Gerstberger R. Nitric Oxide and Body Temperature Control. News Physiol Sci. 1999; 14: 30-36.

28. Gouw Pe. Stimuli affecting exhaled nitric oxide in asthma. Eur Resp Review. 1999; 9: 219-222.

29. Jobsis Q, HC Raatgeep, SL Schellekens, et al. Hydrogen peroxide and nitric oxide in exhaled air of children with cystic fibrosis during antibiotic treatment. Eur Respir J. 2000; 16: 95-100.

30. Bui LT, NI Winters, MI Chung, et al. Chronic lung diseases are associated with gene expression programs favoring SARS-CoV-2 entry and severity. Nat Commun. 2021; 12: 4314.

31. Deng L, A Khan, W Zhou, et al. Follow-up study of clinical and chest CT scans in confirmed COVID-19 patients. Radiol Infect Dis. 2020; 7: 106-113.

32. McGroder CF, D Zhang, MA Choudhury, et al. Pulmonary fibrosis 4 months after COVID-19 is associated with severity of illness and blood leucocyte telomere length. Thorax. 2021.

33. Wang Y, C Dong, Y Hu, et al. Temporal Changes of CT Findings in 90 Patients with COVID-19 Pneumonia: A Longitudinal Study. Radiol. 2020; 296: E55-E64.

34. Mahmud SMH, M Al-Mustanjid, F Akter, et al. Bioinformatics and system biology approach to identify the influences of SARS-CoV-2 infections to idiopathic pulmonary fibrosis and chronic obstructive pulmonary disease patients. Brief Bioinform. 2021; 22.

To cite this article: Lupfer CR, Nadler R, Amen R, et al. Inhalation of Sodium Pyruvate to Reduce the Symptoms and Severity of Respiratory Diseases Including COVID-19, Long COVID, and Pulmonary Fibrosis. European Journal of Respiratory Medicine. 2021; 3:3. 